\title{
Genetics
}

Published online: March 6, 2008 DOI: $10.1159 / 000113697$

\section{Investigating Convergent Actions of Genes Linked to Familial Parkinson's Disease}

\author{
Benjamin Wolozin Shamol Saha Maria Guillily Andrew Ferree Misha Riley \\ Department of Pharmacology, Boston University School of Medicine, Boston, Mass., USA
}

\section{Key Words}

Mitochondria • Rotenone toxicity · Caenorhabditis elegans • Familial Parkinson's disease

\begin{abstract}
Background: Mutations in LRRK2 are among the most frequent genetic changes identified in Parkinson's disease (PD), but how LRRK2 contributes to the pathophysiology of PD is not known. Objectives: To investigate how expressing wildtype or G2019S LRRK2 modifies cellular responses to rotenone, a mitochondrial toxin. Methods: We investigated the vulnerability to mitochondrial toxins in Caenorhabditis elegans expressing wild-type or G2019S LRRK2. Results: We observed a powerful role for LRRK2 in mitochondrial biology. Overexpressing LRRK2 strongly protects $C$. elegans against rotenone toxicity. The G2019S LRRK2 construct also protected LRRK2 against rotenone, but to a lesser degree than wildtype LRRK2. Knockdown of Irk-1 potentiated rotenone toxicity. Conclusions: These data suggest that LRRK $1 / 2$ regulate mitochondrial physiology.

Copyright $\odot 2008$ S. Karger AG, Basel
\end{abstract}

The discovery of genes carrying mutations linked to familial Parkinson's disease (PD) help to identify critical biochemical pathways mediating the disease process. Differing lines of evidence implicate mitochondria, au- tophagy/lysosomes, the ubiquitin proteasomal system, and dopaminergic neurotransmission as cellular targets of damage in models of familial PD. $\alpha$-Synuclein appears to be the predominant fibrillizing protein associated with PD [1]. Parkin, DJ-1 and PINK1 are all associated with autosomal recessive PD. Studies in mice, Caenorhabditis elegans, drosophila and cell culture each show evidence of mitochondrial damage or mitochondrial toxicity $[2,3]$. Disorders of the ubiquitin proteasomal system and of the lysosomal system are implicated based on several lines of study $[4,5]$.

Mutations in LRRK2 are the most common changes associated with familial PD identified to date [6]. Whether LRRK2 interacts with any of the pathways currently implicated in PD, such as dysfunction of mitochondria, the lysosome or dopaminergic function, is currently unknown. The function of LRRK2 is not known, but some biochemical activities are evident from its structure and distribution. LRRK2 is a kinase that is homologous to mixed lineage kinases [6]. The homology to kinases is supported by cell culture studies showing that LRRK2 actively autophosphorylates as well as phosphorylates artificial substrates, such as myelin basic protein $[7,8]$. LRRK2 also contains a domain that is homologous to small GTPases, and is able to bind GTP. LRRK2 kinase activity is implicated in the pathophysiology of PD because most mutations in LRRK2 increase autophosphorylation [7]. Although the physiological target of LRRK2 is unknown,

\section{KARGER}

๑) 2008 S. Karger AG, Basel

Fax +41613061234

E-Mail karger@karger.ch

www.karger.com
Accessible online at:

www.karger.com/ndd
Prof. Benjamin Wolozin, $\mathrm{MD}, \mathrm{PhD}$

Department of Pharmacology, Boston University School of Medicine

715 Albany St., R614

Boston, MA 02118-2526 (USA)

Tel. +1 617414 2652, Fax +1 617414 2651, E-Mail bwolozin@bu.edu 
Fig. 1. LRRK2 modifies vulnerability of C. elegans to rotenone. a NonTG and LRRK2 (wild type) C. elegans were exposed to differing concentrations of rotenone, and survival was analyzed by touch assay after 48 h. b Knockdown of lrk-1 in C. elegans potentiates rotenone-mediated toxicity.

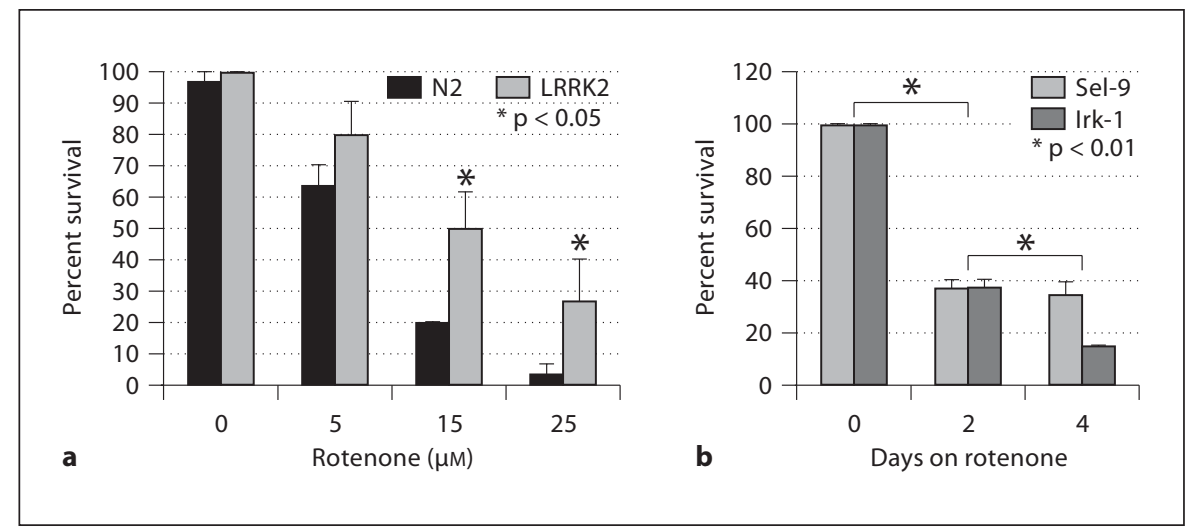

recent observations that LRRK2 is largely membrane associated suggest that it plays a role in the biology of membrane-bound organelles, such as the mitochondria, endoplasmic reticulum and synapse [9]. How these various biochemical activities integrate to affect the biology of neurons remains unknown. In this study, we report on the effects of LRRK2 expression on C. elegans biology.

\section{Methods}

\section{Plasmids}

Wild-type and G2019S LRRK2 genes were inserted into the gateway pENTR $1 \mathrm{~A}$ vectors and sequenced. LRRK2 was then transferred to Lin-15 vectors for C. elegans as described before [10].

\section{C. elegans Culture}

Wild-type and G2019S LRRK2 were driven by the neuronal specific synaptobrevin promoter. The transgenes were integrated into the C. elegans genome by $\gamma$-irradiation, and the worms outcrossed 5 times into an N2 background. Integration was verified by PCR analysis of LRRK2 in the offspring. Viability assays used 3 dishes of 40 adult worms for each line and condition on plates containing NGM agar, OP50 bacteria $\pm 25 \mu \mathrm{M}$ rotenone. After 2 days, the worms were transferred to fresh plates under the same conditions and 2 days later the number of living worms were counted. Viability was determined by touch assay; worms showing no movement of the body or pharynx for $10 \mathrm{~s}$ after being prodded were designated as dead.

\section{Results}

Generation of C. elegans Expressing Wild-Type or G2019S LRRK2

Two lines of wild-type LRRK2 and two lines of G2019S LRRK2 were generated as arrays, integrated and then backcrossed 5 times into Bristol N2 nontransgenic (nonTG) worms. Expression of the transgene was documented by PCR, using amplimers spanning bases 1-330 and 7231-7570 of human LRRK2. Expression of mRNA for LRRK2 was quantified using real-time PCR. mRNA for wild-type and G2019S LRRK2 lines was similar. Gross examination suggests that $C$. elegans expressing wildtype or G2019S LRRK2 was able to develop to adulthood, lay eggs and feed normally. Upon aging, small differences were noted between $C$. elegans expressing wild-type LRRK2, G2019S LRRK2 and those with nonTG lines. C. elegans expressing wild-type LRRK2 exhibited life spans that were approximately $15 \%$ longer than those of C. elegans expressing G2019S LRRK2 or nonTG lines.

To test the vulnerability to mitochondrial toxins, each line of C. elegans was synchronized, grown to the L4 stage and transferred to plates containing NBM agar, OP50 bacteria and $0,5,15$ or $25 \mu \mathrm{M}$ rotenone, which is an inhibitor of complex I of the electron transport chain. After 2 days, the number of viable worms was counted. Worms expressing wild-type LRRK showed significantly less vulnerability to rotenone compared to the nonTG worms (fig. 1a). Parallel experiments compared the vulnerability of worms expressing wild-type and G2019S LRRK2. After 4 days of rotenone treatment, both wild-type and G2019S LRRK2 worms showed more survival than nonTG worms; the relative ratio for survival of the wildtype LRRK2:G2019S LRRK2:N2 (nonTG) worms was 6: 4.5:1 ( $\mathrm{p}<0.0001)$. We also examined the effect of lrk-1 knockdown. Synchronized L4 nonTG worms were fed RNAi feeding bacteria with lrk-1 or sel-9 RNAi vector. Sel-9 serves as a reference gene that does not affect vulnerability to rotenone, based on multiple studies in our laboratory. Two days later, the nematodes were transferred to plates containing the RNAi feeding bacteria and 
$25 \mu \mathrm{M}$ rotenone. Viability was determined by touch assay at 0,2 and 4 days. Knockdown of lrk-1 strongly potentiated rotenone toxicity (fig. 1b).

\section{Discussion}

Mutations in LRRK2 are among the most frequent genetic changes identified in PD, but how LRRK2 contributes to the pathophysiology of PD is not known. In this study, we investigated how expressing wild-type or G2019S LRRK2 modifies cellular responses to rotenone, a mitochondrial toxin. We observed that expressing wildtype or G2019S LRRK2 protects C. elegans against rotenone toxicity. These data suggest that LRRK2 can regulate mitochondrial physiology, possibly via the lrk-1 pathway. The ability of LRRK1/2 to regulate mitochondrial biology is an action shared in common with many genes implicated in PD. Overexpression of Parkin, DJ-1 and PINK1 each protects against mitochondrial toxins. Loss of each of these genes sensitizes to oxidative stresses in cell culture, and knockout of parkin or DJ-1 in drosophila causes age-dependent degeneration of flight muscles. Knockout of these genes in mouse does not produce a strong degenerative phenotype, but does produce abnormal synaptic responses. These data raise the possibility that LRRK2 modulates mitochondrial vulnerability, which is an action that is shared by other PD-related genes.

Analysis of LRRK1 function might also provide an important direction of reference. C. elegans has one LRRK, termed lrk-1, while drosophila and vertebrates have two LRRKs, termed LRRK1 and LRRK2. The function of lrk-1 in C. elegans is not yet understood. We observed that knockdown of lrk-1 potentiates rotenone toxicity in C. elegans, which mirrors the effects observed with LRRK2 expression. Knockdown of LRRK2 also potentiates toxicity in drosophila, which indicates that the role of LRRK2 in cell survival is preserved across species
[11]. One prior publication examined the function of lrk-1 in C. elegans and observed a developmental effect on the distribution of synaptobrevin in a subset of neurons. The more general effect of lrk-1 on vulnerability to rotenone that we observed greatly expands the understanding of lrk-1 function in C. elegans and LRRK2 function generally.

G2019S LRRK2 protected against rotenone toxicity less than wild-type LRRK2. The putative effects of the G2019S mutations could reflect the action of the mutation, but we have not ruled out that the difference arises from dose effects since G2019S LRRK2 is expressed at a higher level than the wild-type LRRK2 constructs. Interpreting any changes in C. elegans requires careful reference to what occurs in the mammalian context. Mutations in LRRK2 associated with familial PD increase kinase activity and cell death of mammalian neurons. These two processes appear to be biochemically linked because transfection studies indicate that LRRK2 kinase activity is required for LRRK2-mediated toxicity. The mutant forms of LRRK2 also cause process retraction in cultured neurons, while knockdown of LRRK2 enhances process outgrowth. These data provide a general picture supporting the hypothesis that disease-related mutations in LRRK2 enhance toxic activity. The direction of change for G2019S LRRK2 in C. elegans is consistent with the increased toxicity observed in mammalian neurons, which suggests that the activities of LRRK2 in C. elegans provide a useful readout for the effects of disease-related mutations. Future studies will need to examine a broader spectrum of mutant constructs to determine whether reduced protection is a constant change associated with disease-related mutations.

\section{Acknowledgements}

This study was supported by grants to B.W. from NIEHS/NIH (ES015567) and the Michael J. Fox Foundation.

\section{References}

1 Spillantini MG, Bird TD, Ghetti B: Frontotemporal dementia and parkinsonism linked to chromosome 17: a new group of tauopathies. Brain Pathol 1998;8:387-402.

$\checkmark 2$ Dauer W, Przedborski S: Parkinson's disease: mechanisms and models. Neuron 2003; 39:889-909.
3 Ved R, Saha S, Westlund B, Perrier C, Burnam L, Sluder A, Hoener M, Rodriguez C, Przedborski S, Alfonso A, Liu L, Wolozin B: Similar patterns of mitochondrial vulnerability and rescue induced by genetic modification of $\alpha$-synuclein, parkin and DJ-1 in Caenorhabditis elegans. J Biol Chem 2005; 280:42655-42668.

\footnotetext{
4 Hardy J, Cai H, Cookson MR, Gwinn-Hardy K, Singleton A: Genetics of Parkinson's disease and parkinsonism. Ann Neurol 2006; 60:389-398.

$\checkmark 5$ Massey AC, Zhang C, Cuervo AM: Chaperone-mediated autophagy in aging and disease. Curr Top Dev Biol 2006;73:205-235.
} 
6 Taylor JP, Mata IF, Farrer MJ: LRRK2: a common pathway for parkinsonism, pathogenesis and prevention? Trends Mol Med 2006; $12: 76-82$.

$\checkmark 7$ West AB, Moore DJ, Choi C, Andrabi SA, Li X, Dikeman D, Biskup S, Zhang Z, Lim KL, Dawson VL, Dawson TM: Parkinson's disease-associated mutations in LRRK2 link enhanced GTP-binding and kinase activities to neuronal toxicity. Hum Mol Genet 2007; $16: 223-232$.
8 Greggio E, Jain S, Kingsbury A, Bandopadhyay R, Lewis P, Kaganovich A, van der Brug MP, Beilina A, Blackinton J, Thomas KJ, Ahmad R, Miller DW, Kesavapany S, Singleton A, Lees A, Harvey RJ, Harvey K, Cookson MR: Kinase activity is required for the toxic effects of mutant LRRK2/dardarin. Neurobiol Dis 2006;23:329-341.

$\checkmark 9$ Biskup S, Moore DJ, Celsi F, Higashi S, West AB, Andrabi SA, Kurkinen K, Yu SW, Savitt JM, Waldvogel HJ, Faull RL, Emson PC, Torp R, Ottersen OP, Dawson TM, Dawson VL: Localization of LRRK2 to membranous and vesicular structures in mammalian brain. Ann Neurol 2006;60:557-569.
10 Clark SG, Lu X, Horvitz HR: The Caenorhabditis elegans locus lin-15, a negative regulator of a tyrosine kinase signaling pathway, encodes two different proteins. Genetics 1994; 137:987-997.

11 Lee SB, Kim W, Lee S, Chung J: Loss of LRRK2/PARK8 induces degeneration of dopaminergic neurons in Drosophila. Biochem Biophys Res Commun 2007;358:534-539. 